\title{
Dialogue social et démocratie en Tunisie dans un contexte de transition
}

\section{Nouri Mzid}

\section{(2) OpenEdition}

1 Journals

\section{Édition électronique}

URL : https://journals.openedition.org/rdctss/1602

DOI : $10.4000 /$ rdctss. 1602

ISSN : 2262-9815

Éditeur

Centre de droit comparé du travail et de la sécurité sociale

\section{Édition imprimée}

Date de publication : 1 avril 2019

Pagination : 7-17

ISSN : 2117-4350

\section{Référence électronique}

Nouri Mzid, "Dialogue social et démocratie en Tunisie dans un contexte de transition », Revue de droit comparé du travail et de la sécurité sociale [En ligne], 1 | 2019, mis en ligne le 01 novembre 2021, consulté le 13 novembre 2021. URL : http://journals.openedition.org/rdctss/1602 ; DOI : https:// doi.org/10.4000/rdctss. 1602

\section{(c) (i) (9)}

Revue de droit comparé du travail et de la sécurité sociale est mise à disposition selon les termes de la Licence Creative Commons Attribution - Pas d'Utilisation Commerciale - Pas de Modification 4.0 International. 


\title{
DiALOGUE SOCIAL ET DÉMOCRATIE EN TUNISIE DANS UN CONTEXTE DE TRANSITION
}

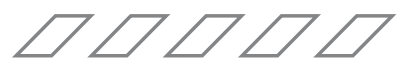

\section{RÉSUMÉ}

La promotion du dialogue social est une exigence de la transition démocratique en Tunisie. Mais, l'efficacité de ce dialogue reste limitée à cause notamment des lacunes de la loi concernant l'institutionnalisation du dialogue social tripartite, ainsi que l'absence d'un cadre juridique adapté en matière de dialogue social bipartite.

MOTS CLÉS: Démocratie, dialogue social, syndicat, relations professionnelles.

\begin{abstract}
Promoting social dialogue is a requirement of the democratic transition in Tunisia. But, the effectiveness of this dialogue remains limited in particular because of the shortcomings of the law concerning the institutionalization of tripartite social dialogue, as well as the lack of an appropriate legal framework for bipartite social dialogue.
\end{abstract}

KEYWORDS: Democraty, Social Dialogue, Trade Union, Industrial relations. 
e dialogue social est une notion centrale dans tout système moderne des relations professionnelles. Ayant pour finalité de pacifier les rapports de travail, il constitue à la fois un instrument clé de promotion de la cohésion sociale et du développement inclusif, ainsi qu'une illustration d'une modernité du système des relations professionnelles ${ }^{1}$. Cette modernité consiste, en effet, à " civiliser » ces relations, c'est-à-dire y substituer aux rapports de forces des rapports de droit à travers des mécanismes de régulation sociale basés sur le dialogue et la concertation.

Le dialogue social est aussi un modèle de gouvernance et une exigence de l'idéal démocratique, voire l'instrument le plus adapté pour la réalisation de la démocratie sociale considérée comme l'indispensable complément de la démocratie politique. Cette affirmation est aujourd'hui, plus que jamais, d'une grande actualité en Tunisie dans un contexte de transition vers la démocratie qui reste encore fragile. Le succès de cette transition dépend dans une large mesure de l'existence d'un véritable consensus social fondé sur le dialogue entre des acteurs représentatifs et légitimes².

L'adoption d'une nouvelle Constitution tunisienne, le 27 janvier 2014, marque une étape décisive dans le processus de cette transition. Elle a largement consolidé les droits sociaux fondamentaux qui constituent une exigence de l'édification de tout Etat social ${ }^{3}$. Mais cette Constitution, comme celle de 1959, a totalement ignoré le principe du dialogue social et la liberté de négociation collective qui constitue son instrument essentiel. Pourtant, cette liberté est un prolongement indissociable du principe de la liberté syndicale, qui est solennellement proclamé par la Constitution ${ }^{4}$ et largement consacré par le Code du travail ${ }^{5}$.

1 Voir M. Tarchouna, "La modernisation du système tunisien des relations professionnelles, bilan et perspective », in Tunisie 2040 : le renouvellement du projet moderniste tunisien, Ouv. coll., Association Mohamed Ali de la culture ouvrière, Sud Editions, Tunis 2011, p. 415.

2 Voir Institut international d'études sociales, Tunisie, un nouveau contrat social pour une croissance juste et équitable, BIT,2011. Sur le rôle des syndicats dans la transition démocratique en Tunisie, v. N. Mzid (dir.), Syndicats et transition démocratique, Etudes à la mémoire du Doyen M. Tarchouna, Faculté de droit de Sfax, 2016. V. aussi N.Mzid et A. Hamza Safi, « Droit du travail et transition démocratique en Tunisie », in Mélanges en l'honneur d'Antoine LyonCaen, Dalloz, 2018, p. 693.

3 Voir notre article intitulé "L'insertion des droits sociaux fondamentaux dans la nouvelle Constitution tunisienne : une effectivité à l'épreuve », Revue de droit comparé du travail et de la sécurité sociale, 2015/1, p. 80.

4 Affirmé par la Constitution du $1{ }^{\text {er }}$ juin 1959 (art. 8), le principe de la liberté syndicale a été consolidé par la Constitution du 27 janvier 2014 (art. 35 et 36).

5 Voir le chapitre 1 du Livre VII du C.T, intitulé : Les syndicats professionnels. 
Le principe du dialogue social trouve son fondement, surtout, dans un ensemble de normes adoptées par l'OIT et ratifiées par la Tunisie ${ }^{6}$. Fondée sur le principe du tripartisme, cette organisation encourage largement les pays membres à promouvoir la coopération entre les autorités publiques et les organisations d'employeurs et de travailleurs, à travers le recours au dialogue social tripartite, ainsi qu'entre ces organisations à travers le recours au dialogue social bipartite ${ }^{7}$. Mais il n'existe pas un modèle universel de dialogue social. Les instruments de l'OIT relatifs à ce sujet laissent aux Etats membres une grande latitude quant aux modalités et procédures à adopter en la matière.

En Tunisie, la pratique du dialogue social, notamment sous sa forme de négociation collective, est relativement enracinée dans le système des relations professionnelles. Mais l'efficacité de ce système reste souvent limitée, pour des raisons multiples dont l'absence d'une organisation juridique claire permettant de garantir la vitalité et la continuité du dialogue social et de renforcer son efficience. Cette affirmation peut être vérifiée, d'abord, au niveau du dialogue social tripartite s'exerçant à l'échelle nationale entre les représentants du gouvernement et des organisations des salariés et des employeurs. L'institutionnalisation de cette forme de dialogue, à travers la création d'un organe spécifique, dénommé le conseil national du dialogue social (CNDS), par la loi n 54 du 24 juillet 2017, peut être un appui à la transition vers la démocratie. Mais l'apport de cette loi reste mitigé, étant donné ses lacunes et ambiguïtés qui risquent d'affecter le rôle de cet organe et les modalités de son fonctionnement (I). Ensuite, un manque d'efficacité du système tunisien des relations professionnelles peut être observé au niveau du dialogue social bipartite. En effet, le dispositif relatif aux structures de ce dialogue au sein de l'entreprise reste inadapté, et le cadre juridique dans lequel s'exerce la principale forme de ce dialogue, à savoir la négociation collective normative, est très lacunaire (II).

6 Parmi ces normes, on peut mentionner notamment la convention $n^{\circ} 87$ sur la liberté syndicale et la protection du droit syndical, et la convention $n^{\circ} 98$ sur le droit d'organisation et de négociation collective. Ces deux conventions qui font partie du bloc des normes fondamentales du travail ont été ratifiées par la Tunisie depuis 1957. La Tunisie a ratifié aussi, en 2007, la convention de l'OIT n 135 sur la représentation des travailleurs dans l'entreprise. De même, la Tunisie a ratifié, en 2013, trois autres conventions de I'OIT, ayant un rapport étroit avec le principe du dialogue social, à savoir : la convention $n^{\circ} 144$ sur le dialogue social tripartite, la convention $n^{\circ} 151$ sur les relations du travail dans la fonction publique et la convention $\mathrm{n}^{\circ} 154$ sur la négociation collective.

7 Voir BIT, Dialogue social, Conférence internationale du travail, 102 ${ }^{\mathrm{ème}}$ session, 2013. 


\section{I - L'INSTITUTIONNALISATION DU DIALOGUE SOCIAL TRIPARTITE, UN APPUI MITIGÉ À LA TRANSITION VERS LA DÉMOCRATIE}

Le législateur tunisien avaitcréé, en 1996, une structure dénommée «la commission nationale du dialogue social ", qui était chargée d'émettre son avis sur diverses questions concernant notamment la législation du travail, les normes internationales du travail, les salaires, la classification professionnelle, les négociations collectives et le climat social ${ }^{8}$. Mais cette structure a connu un échec total. Ne disposant pas d'une réelle autonomie vis-à-vis des autorités publiques et ayant une composition dominée par les représentants du gouvernement, elle a été boycottée par la centrale syndicale UGTT 9 . Une réforme de la loi, en vue de promouvoir et d'institutionnaliser le dialogue social tripartite, est devenue alors d'une grande nécessité, comme l'ont exprimé les signataires du contrat social en $2013^{10}$. Cette réforme a été concrétisée par la loi $n^{\circ} 54$ du 24 juillet 2017 portant création du conseil national du dialogue social. Mais l'analyse de cette loi permet de constater que si le champ d'intervention de cette nouvelle structure est très étendu $(A)$, son fonctionnement reste mal encadré (B).

\section{A - UN CHAMP D'INTERVENTION ÉTENDU}

Le CNDS est appelé à assurer des fonctions multiples se rapportant essentiellement à deux grands domaines d'intervention. D'abord, il est chargé d'une fonction d'organisation et de gestion du dialogue social. Dans ce cadre, la loi a confié au Conseil plusieurs missions qui semblent ambitieuses, visant notamment la promotion du dialogue social en assurant sa continuité et sa régularité, la pacification des relations professionnelles, l'encadrement des négociations collectives, la présentation des propositions concernant l'évolution du salaire minimum garanti, ainsi que l'instauration d'un climat social paisible garantissant les conditions du travail décent et encourageant à l'investissement.

Mais ces missions restent parfois mal définies et formulées dans des termes très vagues. Ainsi, à titre d'exemple, tout en chargeant le CNDS de « garantir un dialogue social tripartite efficient ", la loi n'a fixé aucun critère objectif et précis permettant de mesurer l'impact de l'intervention du Conseil dans ce domaine et d'évaluer son efficience. De même, la mission d'encadrement des négociations collectives est très vague. S'agit-il d'un simple rôle de facilitation et d'assistance des partenaires sociaux en vue d'assurer un bon déroulement des négociations, ou s'agit-il d'exercer un pouvoir d'organisation et de contrôle du processus des négociations collectives, englobant à la fois l'habilitation des agents de négociation ainsi que la fixation de la périodicité des négociations et leurs modalités procédurales?

8 Voir l'article 335 (ancien) du C.T.

9 Union générale tunisienne du travail.

10 Voir le contrat social signé en date du 14 janvier 2013 entre le gouvernement, I'Union générale tunisienne du travail et l'Union tunisienne pour l'industrie, le commerce et l'artisanat (disponible sur internet). 
Ensuite, le CNDS est impliqué dans la gouvernance des affaires publiques, en formulant ses avis sur l'élaboration des réformes et des politiques de l'Etat dans le domaine économique et social. Cette fonction englobe aussi des aspects multiples, qui se traduisent notamment par la consultation obligatoire du Conseil sur les projets de lois et de décrets gouvernementaux ayant trait au travail, aux relations professionnelles, à la formation professionnelle et à la protection sociale.

Le domaine de la consultation obligatoire couvre aussi l'intervention du Conseil pour exprimer son avis sur la ratification des conventions internationales du travail et contribuer à l'élaboration et à la rédaction des rapports présentés par le gouvernement à l'OIT. Cette mission spécifique qui s'inscrit dans l'application des dispositions de la Convention internationale du travail $n^{\circ} 144$, ratifiée par la Tunisie en 2013, est une illustration du principe du tripartisme qui gouverne le fonctionnement de I'OIT et ses activités normatives, et qui exige d'être conforté au niveau national par un dialogue tripartite en vue de promouvoir la ratification et l'application effective des normes internationales du travail.

En dehors du domaine de la consultation obligatoire, le Conseil peut également être consulté, à titre facultatif, sur les projets de lois et des décrets gouvernementaux à caractère économique et social, ainsi que sur les plans de développement et les budgets économiques.

Dans tous les cas, le Conseil émet son avis dans un rapport motivé qu'il transmet au chef du gouvernement dans un délai d'un mois à compter de sa réception du projet du texte qui lui est soumis. Cet avis est obligatoirement annexé aux projets de loi soumis au Parlement. Mais, juridiquement, le gouvernement peut toujours opter pour un passage en force en cas de retard ou de blocage concernant la formulation des avis du Conseil.

Par ailleurs, le législateur a donné au Conseil, par la technique d'auto-saisine, la possibilité d'être l'initiateur des projets de réforme. En réalité, cette technique a un domaine d'application limité aux questions ayant trait au travail et aux relations professionnelles. Mais, elle revêt une importance dans la mesure où elle permet au Conseil de déclencher le processus des réformes sur des questions dont il juge l'intérêt de soulever et de présenter des propositions à leur sujet aux autorités compétentes. Certes, ces autorités ne sont pas tenues par un délai précis pour examiner ces propositions, ni de les approuver. Mais, pour donner une crédibilité au dialogue social tripartite dans ce domaine, les autorités concernées devraient étudier sérieusement les propositions du Conseil dans des délais raisonnables, avant de décider des suites à leur donner.

Tout en disposant d'un champ d'intervention étendu, le CNDS risque de voir son rôle limité à cause des faiblesses relatives aux modalités de son fonctionnement.

\section{B - UN FONCTIONNEMENT MAL ENCADRÉ}

Plusieurs faiblesses risquent d'affecter les modalités de fonctionnement du CNDS. D'abord, une certaine ambiguïté peut être signalée concernant la représentativité syndicale au sein du Conseil. L'article 8 de la loi du 24 juillet 2017 dispose que cette structure "se compose d'un nombre égal de représentants du 
gouvernement, de représentants des organisations les plus représentatives des travailleurs et de représentants des organisations les plus représentatives des employeurs dans les secteurs agricole et non agricole ». En utilisant le pluriel à propos des organisations des travailleurs et des employeurs, le législateur adopte ici une conception pluraliste de la représentativité syndicale au sein du Conseil. Or, les textes réglementaires d'application de la loi ${ }^{11}$ ont retenu une conception majoritaire de cette représentativité ${ }^{12}$, en se référant à « l'organisation la plus représentative » des travailleurs et des employeurs. Un problème de légalité des textes mentionnés peut alors être évoqué, à cause de leur manque de conformité à la loi à ce sujet.

De même, la loi a gardé le silence concernant les critères de la représentativité syndicale au sein du CNDS. C'est l'arrêté du Ministre des affaires sociales du 26 septembre 2018 qui est venu fixer ces critères. Selon son article 2, " est considérée l'organisation syndicale des travailleurs et des employeurs la plus représentative, l'organisation qui a tenu son congrès électoral et celle qui a le plus grand nombre d'adhérents et le plus grand nombre des structures sectorielles, régionales et locales ». Parmi ces critères, celui du nombre des adhérents apparaît comme étant le plus significatif, voire le critère décisif de la représentativité syndicale. Mais les autres critères mentionnés par l'arrêté semblent être des critères formels et insuffisants à mesurer l'audience de l'organisation syndicale, son influence, ainsi que son indépendance et son attachement aux valeurs de la démocratie.

Par ailleurs, le législateur a prévu d'impliquer dans l'assemblée générale du Conseil d'autres membres désignés en leur qualité, ainsi que des membres reconnus pour leur expérience et leur compétence dans les domaines juridiques, sociaux et économiques. Mais, la loi n'a pas fixé de critères précis pour la désignation de ces membres ${ }^{13}$. En revanche, le législateur n'a pas prévu la possibilité d'impliquer d'autres corps de la société civile, à côté des représentants des organisations syndicales des travailleurs et des employeurs, telles que les associations de défense des chômeurs. Pourtant, la participation de telles composantes de la société civile peut être très utile pour renforcer le rayonnement du Conseil et tenir compte d'une plus large diversité des intérêts en jeu.

D'autres faiblesses peuvent être constatées, ensuite, au niveau de l'organisation interne du CNDS. La loi du 24 juillet 2017 dispose, dans son article 1er, que le Conseil " est doté de l'autonomie administrative et financière ", mais sans indiquer explicitement qu'il jouit de la personnalité morale. En réalité, un manque d'autonomie

11 Voir le décret $n^{\circ} 676$ du 7 août 2018 portant fixation du nombre des membres du CNDS, et l'arrêté du Ministre des affaires sociales en date du 26 septembre 2018 portant fixation des critères pour déterminer l'organisation syndicale la plus représentative à l'échelle nationale pour la composition du CNDS.

12 Sur la distinction entre la conception pluraliste et la conception majoritaire de la

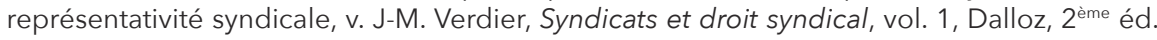
1987, p. 480.

13 D’ailleurs, le décret du 7 août 2018 a totalement gardé le silence concernant ce sujet. 
du Conseil peut être observé à travers quelques dispositions de la loi. Ainsi, le budget du Conseil relève de celui du Ministère des affaires sociales. Son règlement intérieur doit être approuvé par décret gouvernemental. De même, l'organisation administrative et financière du Conseil est fixée par décret gouvernemental. Du reste, sa gestion administrative et financière est assurée par un directeur désigné par décret gouvernemental. Tous ces éléments risquent d'affaiblir considérablement l'autonomie du Conseil et d'affecter l'efficacité de son fonctionnement et sa crédibilité.

Par ailleurs, des lacunes peuvent être signalées aussi au niveau des mécanismes d'intervention du Conseil. Ainsi, tout en instituant une obligation de consulter le CNDS dans plusieurs domaines déjà mentionnés, le législateur n'a pas indiqué la sanction en cas de défaillance à cette obligation. De même, aucune modalité n'a été prévue par la loi pour assurer un meilleur impact des avis et des propositions exprimés par le Conseil aux autorités compétentes sur les décisions finales à adopter par ces dernières. Ce vide juridique peut limiter l'efficacité des attributions du Conseil et engendrer un manque d'articulation entre la démocratie sociale, à travers les mécanismes du dialogue tripartite, et la démocratie politique.

Enfin, le CNDS en tant qu'organe consultatif n'est pas investi d'un pouvoir normatif analogue à celui accordé aux syndicats représentatifs comme agents de la négociation collective. Mais l'absence d'une délimitation précise de son rôle en matière d'encadrement des négociations collectives peut engendrer aussi un problème d'articulation entre le dialogue social tripartite et le dialogue social bipartite à cause du risque d'empiétement du Conseil sur le pouvoir des syndicats dans le domaine de la négociation collective.

\section{II - L'INDISPENSABLE MODERNISATION DU DIALOGUE SOCIAL BIPARTITE, UNE EXIGENCE DE L'IDÉAL DÉMOCRATIQUE}

La démocratisation des rapports de travail passe essentiellement à travers la promotion du dialogue social bipartite, ce qui exige le renforcement du rôle des organisations de salariés et d'employeurs en tant qu'acteurs incontournables de la démocratie sociale. En effet, sans la liberté de dialogue et de négociation entre interlocuteurs représentatifs et légitimes, il est vain de parler de démocratie sociale. Mais, le système tunisien des relations professionnelles souffre à ce niveau de plusieurs insuffisances qui limitent considérablement l'efficacité du dialogue social. D'une part, le dispositif relatif aux structures de ce dialogue au niveau de l'entreprise est inadapté (A). D'autre part, le cadre juridique dans lequel s'exerce la principale forme de ce dialogue, à savoir la négociation collective, est encore lacunaire (B). 


\section{A - LES STRUCTURES DU DIALOGUE SOCIAL DANS L'ENTREPRISE : UN DISPOSITIF INADAPTÉ}

En Tunisie, le dialogue social dans l'entreprise repose sur un système dualiste de représentation des salariés, mais qui est marqué par une grande ambivalence ${ }^{14}$. Alors que les organes de représentation non syndicale, qui sont institués par la loi, ont fait preuve de leur inefficacité comme structures de dialogue social, la présence de l'organe syndical dans l'entreprise, qui s'impose en réalité comme étant le principal interlocuteur du chef d'entreprise au nom des salariés, est encore quasi ignorée par le Code du travail.

Le législateur a prévu, depuis 1960, l'institution du comité d'entreprise en tant qu'organe de représentation élu du personnel. Mais cette structure n'a pas connu un grand succès et a été remplacée, depuis 1994, par une autre structure dénommée la commission consultative d'entreprise ${ }^{15}$. Contrairement à l'ancien comité d'entreprise qui était composé seulement par des représentants élus du personnel, cette commission est une structure paritaire composée par un nombre égal de représentants désignés par la direction de l'entreprise et de représentants des travailleurs élus par ces derniers. De part sa composition paritaire et la multiplicité des domaines de son intervention ${ }^{16}$, elle semble avoir une vocation comme structure de dialogue et de concertation au sein de l'entreprise. Mais, en réalité, il n'est pas difficile de constater que le système de représentation élue du personnel est toujours en crise, pour plusieurs raisons. D'abord, ce système ne concerne qu'un nombre très faible d'entreprises, étant donné que le tissu économique tunisien est composé majoritairement par des entreprises de très petite taille, qui ne répondent pas à la condition d'effectif exigée par la loi pour la mise en place des structures de représentation du personnel. Ensuite, les attributions de la commission consultative d'entreprise, notamment en matière économique, demeurent très vagues. Sur ce plan, on peut observer un grand recul par rapport à la législation relative à l'ancien comité d'entreprise, selon laquelle celuici « est consulté sur les questions intéressant l'organisation de l'entreprise, de manière à être associé progressivement à sa gestion et à son développement ${ }^{17}$. Du reste, les

14 Sur ce système dualiste de représentation des salariés dans l'entreprise en droit tunisien, v. notre thèse, La liberté syndicale en droit tunisien, Faculté de droit et des sciences politiques de Tunisie, 1995, p. 297.

15 Art. 157 et s. du C.T. Cette structure doit être créée dans toute entreprise employant au moins quarante salariés permanents. Dans les entreprises employant un nombre de salariés permanents égal ou supérieur à vingt et inférieur à quarante, la loi prévoit l'élection d'un délégué titulaire du personnel et un délégué suppléant.

16 La Commission consultative d'entreprise intervient dans divers domaines liés à la gestion du personnel, dont notamment l'organisation du travail, la gestion des œuvres sociales, la formation professionnelle, la santé au travail, et la discipline des salariés (art. 160 du C.T). Du reste, elle est chargée aussi d'intervenir comme structure de conciliation en cas de conflits collectifs de travail au sein de l'entreprise (art. 376 du C.T).

17 Ancien art. 161 du C.T 
moyens dont dispose la commission consultative d'entreprise pour l'exercice de ses fonctions sont très limités. D'ailleurs, la commission n'est pas dotée de la personnalité juridique et ne dispose pas d'un budget propre pour son fonctionnement. De ce point de vue, il n'est pas excessif d'affirmer qu'elle reste dans une dépendance totale à l'égard de l'employeur. Tous ces facteurs ne peuvent que provoquer le désintérêt des salariés et la grande méfiance des syndicats à l'égard de cette commission qui est devenue, de fait, une instance exclusivement disciplinaire, et dont l'échec dans sa fonction comme structure de dialogue social ne fait aucun doute ${ }^{18}$.

En revanche, c'est l'organe syndical qui exerce effectivement cette fonction et s'impose comme étant le principal organe intervenant en tant que porte-parole des salariés et interlocuteur du chef d'entreprise. Mais sa présence sur les lieux du travail reste sous le signe de la précarité à cause d'une attitude équivoque du législateur. En effet, tout en proclamant le principe de la liberté syndicale, garanti par la Constitution ${ }^{19}$ et en accordant aux syndicats des prérogatives très étendues, leur permettant d'intervenir comme acteur principal du dialogue social sur le plan national et collaborateurs de l'Etat à l'organisation de la profession, le Code du travail garde un silence quasi-total s'agissant de la place du syndicat dans l'entreprise. II ne réglemente pas, ainsi, sa présence sur les lieux du travail et ne lui accorde pas des prérogatives spécifiques à ce niveau comme structure de représentation du personnel. Le législateur semble toujours ignorer le rôle du syndicat comme organe d'expression de l'identité collective des salariés au sein de l'entreprise.

Cette abstention du législateur n'a pas empêché l'organe syndical de s'affirmer dans plusieurs entreprises où il est devenu un acteur incontournable pour tout dialogue social sérieux et authentique. Ce rôle est confirmé par les conventions collectives qui ont introduit depuis 1973 l'ébauche d'un statut du syndicat dans l'entreprise, en tant que porte-parole des salariés et interlocuteur privilégié du chef d'entreprise. Elles ont créé, ainsi, à la charge de celui-ci l'obligation de reconnaître l'organisation syndicale représentée par ses délégués à l'entreprise et de recevoir ces derniers une fois par mois et toutes les fois qu'il y a urgence ${ }^{20}$.

Mais la voie conventionnelle, qui reste par définition une source fragile et souvent déterminée par le rapport des forces entre les partenaires sociaux, est-elle suffisante pour encadrer juridiquement l'exercice d'un droit aussi fondamental que celui relatif à l'action syndicale dans l'entreprise? Rien n'est moins sûr, d'autant plus que la Constitution tunisienne dispose dans son article 65 que les textes régissant le statut des syndicats doivent prendre la forme d'une loi organique. Une intervention

18 Voir H. Kotrane, 50 ans après. Plaidoyer pour un nouveau Code du travail, Cahier de l'ITES, $n^{\circ} 3$, mars 2017, p. 79. Du même auteur, «Quel modèle de dialogue social en droit du travail tunisien ?", in Consensus social : discours et pratiques, ouv. coll., ARFORGHE et KonradAdenauer-Stiftung, Tunis, 2006, p. 133.

19 Art. 35 et 36 de la Constitution du 27 janvier 2014.

20 Sur l'apport des conventions collectives concernant la présence du syndicat dans l'entreprise en droit tunisien, v. notre th. précitée, p. 216. 
du législateur en vue d'introduire une réforme substantielle du droit syndical dans l'entreprise nous semble alors nécessaire. Cette réforme peut contribuer à la réconciliation entre le syndicat et l'entreprise et favoriser l'émergence d'une nouvelle dynamique du dialogue social au sein de celle-ci, notamment à travers la promotion de la négociation collective.

\section{B - LA NÉGOCIATION COLLECTIVE COMME PROCESSUS DE DIALOGUE SOCIAL : UN CADRE JURIDIQUE LACUNAIRE}

La négociation collective est un instrument privilégié du dialogue social bipartite. Elle occupe une place essentielle en tant que technique d'encadrement normatif des rapports de travail. Grâce à cette fonction, on assiste à l'émergence d'un droit négocié, comme moyen de régulation sociale, à côté du droit étatique. C'est l'une des manifestations les plus importantes de la démocratie sociale.

Dans sa conception moderne, l'institution de la négociation collective n'est plus conçue seulement sous l'angle de ses résultats enregistrés sous forme d'accords collectifs conclus entre les parties concernées. Elle est saisie aussi en tant que processus qui nécessite d'être encadré juridiquement dans ses différentes étapes. Ainsi, le droit de la négociation collective est devenu essentiellement un droit procédural qui conditionne largement les résultats des négociations consignés dans des accords collectifs. Or, le droit tunisien est très en retard par rapport à cette conception moderne de l'institution de la négociation collective. Cela tient à l'absence d'un cadre juridique adéquat relatif à l'organisation du processus de la négociation ${ }^{21}$. Ce vide juridique se manifeste, d'abord, au niveau de la représentativité des syndicats habilités à négocier et conclure les conventions collectives. Dans un contexte marqué par l'émergence d'un pluralisme syndical, qui trouve son assise juridique dans le principe de la liberté syndicale garantie par la Constitution, ce vide juridique est aujourd'hui une source de surenchère syndicale et de tension sociale.

En réalité, le législateur tunisien n'ignore pas totalement la notion de représentativité syndicale, notamment en matière de négociation collective. II retient, ainsi, la notion d'organisations syndicales les plus représentatives auxquelles il attribue un monopole dans la négociation et la conclusion des conventions collectives. Mais, la loi ne fixe pas les critères d'appréciation de cette représentativité, ni son régime juridique. A cet égard, l'article 39 du Code du travail se contente de signaler qu'en cas de différend au sujet de la représentativité syndicale, un arrêté du Ministre chargé des affaires sociales déterminera les organisations syndicales habilitées à conclure la convention collective. Une intervention du législateur pour fixer des critères objectifs et précis de la représentativité syndicale dans ce domaine est aujourd'hui d'une grande nécessité afin d'éviter tout risque de partialité ou d'abus de la part de l'autorité publique 22 .

21 Cf. notamment, M. Tarchouna, La négociation collective en Tunisie, thèse, Université de Paris 1, 1986.

22 Les critères de représentativité syndicale, tels que fixés par l'arrêté du Ministre des affaires sociale en date du 26 septembre 2018, ne concernent que la détermination des organisations syndicales habilitées à être membre du Conseil national du dialogue social. Voir supra. 
Le législateur est appelé aussi à clarifier sa conception en matière de représentativité syndicale : s'agit-il d'une conception fondée sur le principe d'exclusivité qui attribue un monopole de négociation à l'organisation déclarée la plus représentative, ou d'une conception proportionnelle qui permet d'attribuer la qualité d'organisation représentative à deux ou plusieurs syndicats ? De même, le législateur est appelé à préciser à quel niveau doit être appréciée la représentativité des syndicats : au niveau national, celui de la branche, celui de l'entreprise, ou encore à tous ces niveaux de manière combinée?

Un autre vide juridique concerne l'organisation institutionnelle des négociations collectives $^{23}$. Ce vide juridique est la conséquence de l'absence d'un droit de la négociation collective, et plus particulièrement de l'absence d'une obligation de négocier. De même, le droit tunisien ne fixe pas la périodicité des négociations, ni les modalités et les procédures concrètes relatives à leur déroulement et à la révision des conventions collectives de travail. Toutes ces lacunes font que la négociation collective risque d'être soumise à l'affrontement des acteurs sociaux et à la loi du plus fort, et de perdre ainsi une grande partie de sa signification et de sa dynamique.

Du reste, les négociations collectives en Tunisie sont très centralisées au niveau national et celui de la branche. II en résulte que la négociation d'entreprise n'occupe qu'une place subsidiaire et reste cantonnée dans un rôle mineur. D'ailleurs, l'attitude du législateur ne favorise pas le développement de la négociation d'entreprise. La loi établit, en effet, une hiérarchie rigide qui donne à la négociation de branche une place centrale par rapport à la négociation d'entreprise. Ainsi, conformément à l'article 44 du Code du travail, la convention d'entreprise ne peut être conclue que dans le cadre d'une convention de branche applicable à cette entreprise, sauf dérogation spéciale du ministre des affaires sociales. En plus, la convention d'entreprise ne peut, en aucune façon, contenir des dispositions moins favorables pour les salariés que celles de la convention de branche applicable à l'entreprise. Le contenu de la convention d'entreprise reste, alors, largement conditionné par celui de la convention de branche. Le législateur a voulu, ainsi, éviter une fragmentation des conditions de travail et d'emploi. Mais ce choix du législateur a abouti à une centralisation excessive de la négociation collective et à sa méconnaissance de la situation réelle de l'entreprise.

Par ailleurs, la négociation collective en Tunisie reste largement centrée sur la question salariale, au détriment d'autres questions qui revêtent aujourd'hui une grande importance, telles que les questions relatives à la gestion prévisionnelle de l'emploi, à la formation professionnelle, à la démocratisation des rapports de travail au sein de l'entreprise, à la productivité et à l'intéressement des salariés aux résultats de l'activité. Or, la négociation relative à ces questions ne peut s'accommoder qu'avec le niveau de l'entreprise. D'où la nécessité d'une diversification de la structure et du contenu de la négociation collective pour qu'elle soit mieux adaptée à la réalité mouvante des rapports socioprofessionnels et puisse jouer pleinement sa fonction comme instrument privilégié de dialogue social.

23 Cf. M. Tarchouna, thèse précitée. Du même auteur, « Négociation collective et système de relations professionnelles, quel consensus ?", in Consensus social : discours et pratiques, op. cit., p. 149. 


\section{DiALOgUE SOCIAL ET DÉMOCRATIE EN TUNISIE}

Pour conclure, une intervention du législateur en vue d'introduire une réforme substantielle du droit de la négociation collective semble nécessaire. Mais elle doit s'inscrire dans une démarche globale de modernisation du système des relations professionnelles, permettant d'introduire une nouvelle dynamique du dialogue social. Sans cette réforme, l'avènement de la démocratie sociale restera du domaine de l'utopie.

\section{NOURI MZID}

Professeur agrégé et ancien Doyen de la Faculté de droit de l'Université de Sfax (Tunisie).

Thèmes de recherche : Droit du travail, droit de la sécurité sociale, libertés et droits fondamentaux.

\section{Publications:}

" Droit du travail et transition démocratique en Tunisie " (avec Aicha Hamza Safi), in Mélanges en l'honneur d'Antoine Lyon-Caen, Dalloz, 2018, p. 693.

"L'incitation à l'emploi : quoi de neuf ? ", in L'incitation à l'investissement, quoi de neuf ?, Revue Tunisienne de Fiscalité, $\mathrm{n}^{\circ} 27,2018$, p. 305.

"Le procès en droit social ", in Le procès, Actes du colloque organisé en I'honneur de Maître Najib Féki, Faculté de droit de Sfax, 2017, p. 21. 